Revue d'histoire de l'Amérique française

RAS REVUE D.HISTOIRE DE L'AMÉRIQUE FRANÇAISE

\title{
Un cours de civilisation canadienne-française aux États-Unis
}

\section{Marine Leland}

Volume 2, numéro 2, septembre 1948

URI : https://id.erudit.org/iderudit/801452ar

DOI : https://doi.org/10.7202/801452ar

Aller au sommaire du numéro

Éditeur(s)

Institut d'histoire de l'Amérique française

ISSN

0035-2357 (imprimé)

1492-1383 (numérique)

Découvrir la revue

Citer cet article

Leland, M. (1948). Un cours de civilisation canadienne-française aux États-Unis. Revue d'histoire de l'Amérique française, 2(2), 213-229.

https://doi.org/10.7202/801452ar d'utilisation que vous pouvez consulter en ligne.

https://apropos.erudit.org/fr/usagers/politique-dutilisation/ 
Civilisation CanadienNe-Francaise. - Nous publions dans ce no de la Revue, Un cours de civilisation canadienne-française, article de l'un de nos membres-correspondants, Mlle Marine Leland. Les lignes qui vont suivre et que nous empruntons à la Revue de l'Université Laval, renseigneront nos lecteurs, s'il en est besoin, sur l'opportunité d'un tel cours et sur le mérite de Mlle Leland, véritable initiatrice en ce domaine.

( Il est évident que toute Université digne de ce nom devra bientôt offrir un cours, au Canada, sur la civilisation et la littérature canadiennesfrançaises aux points de vue: (1) social, (2) politique, (3) littéraire, (4) religieux; je dis religieux car, à mon avis, il est possible de s'approcher de ce sujet qui est, comme je l'ai dit au début, inséparable de l'éducation. A mon humble avis,sl l'on veut enseigner un cours sur le Canada français, dans une Unıversité, on ne peut le faire seulement au point de vue littéraire. Si le professeu: n'a pas suivi les cours d'une Université canadienne-française, il est fort probable que ses bonnes intentions feraient plus de mal que de bien, au Canada »)

(Extrait de La Revue de l'Université Laval, Québec, juin 1948, vol. II - no 10. "A la recherche de la vérité canadienne " par Frédéric J. L. Bronner, Hamilton, Ont.) 


\title{
UN COURS \\ DE CIVILISATION CANADIENNE-FRANÇAISE AUX ETATS-UNIS ${ }^{1}$
}

\author{
HISTORIQUE DU COURS
}

\begin{abstract}
A sa réunion de mai 1940, la Faculté de Smith College approuvait avec d'autres projets de cours que Jui soumettait la Doyenne, Dr. Marjorie Hope Nicolson, la fondation d'un cours de semestre sur la littérature canadienne-française. ${ }^{2}$ Depuis cette date, le cours a été
\end{abstract}

1. On comprendra que je n'aie pas pris sur moi de décrire ici certains autres cours sur le Canada françai : qui sont professés aux États-Unis; tels celui que fait M. Antoine Jobin à l'Université de Michigan ou celui qu pro essait à Northwestern, $\mathbf{M}$. J.-M. Carrière qui est actuellement à l'Université de Virginie.

2. En 1942, Miss Nicolson quittait Smith College pour accepter une chaire de littérature anglaise dans la Graduate School de l'Université Columbia. La première femme à être nommée professeur agrégé de la Graduate School, elle est actuellement le chef de cette faculté (section anglaise) et elle fait partie du Conseil de l'université. Je tiens particulièrement à remercier ici Miss Nicolson de l'intérêt qu'elle manifesta à l'égard du cours que je désirais établir lors de la toute première entrevue que j'eus avec elle à ce sujet. Ses sympathiques encouragements et son intelligence des rouages administratifs furent des facteurs décisifs dans la fondation du cours qui fait l'objet de cet article.

Le suecès de cette entreprise fut également assuré par l'aide que lui prêtèrent d'au tres personnalités à Smith College et en dehors de cette institution. Parmi celles-là, je mentionne avec un sentiment de reconnaissance toute spéciale, Miss Annetta I. Clark, Secrétaire de la Board of Trustees of Smith College, qui, tout au cours d'une longue carrière administrative, n'a jamais ménagé son aide efficace aux membres de la Faculté. Grâce à elle, il me fut possible de réaliser plusieurs projets dont l'exécution eat été impossible sans son intervention.

Des encouragements très substantiels me vinrent aussi de l'extérieur. De 1941 à 1944, Mr. Walter S. Rogers, Directeur de l'Institut of Current World Affairs, me fournit les subsides nécessaires pour faire de fréquents voyages au Canada qui me permirent de me documenter sur place. Grâce à sa générosité, je pus me rendre jusqu'à la Rivière la. Paix et prendre contact avec les Canadiens françai s de l'Ouest. Guère n'est besoin d'insister sur l'enrichissement qu'apportèrent à mon enseignı ment ces expériences inestimables.

$\mathrm{Si}$ la liste de mes compatriotes à qui je suis infiniment redevable des marques d'intérêt qu'ils ont portées au cours de civilisation canadienne-française est trop 
professé régulièrement chaque année, et, bien entendu, il a évolué. La matière qui y est traitée ne se borne plus à la littérature. Elle inclut quelques notions sur le fonds géographique, historique, politique et économique que reflète toute littérature. A la suite de cette évolution, le nom du cours a également changé. Celui-ci s'intitule actuellement: French Canadian Civilization et il est inscrit au catalogue sous la rubrique: French 312 a. Il s'adresse à des étudiantes de $3 \mathrm{e}$ et $4 \mathrm{e}$ années et il appartient, comme il l'a toujours fait, au groupe de cours qu'on nomme dans les universités américaines "electives " (cours facultatifs) par opposition aux "required courses" (cours obligatoires) que les élèves doivent suivre pour obtenir leur B.A.dans un champ de spécialisation quelconque. Ces cours facultatifs sont répartis dans une foule de domaines. Ils présentent l'avantage de permettre aux étudiants d'élargir et d'enrichir leur culture en s'initiant, tout au moins, à l'étude de matières qui sont ou en dehors de celles dans lesquelles ils se spécialisent ou qui ne s'y rattachent que d'une façon indirecte. En vue du nombre considérable de cours facultatifs qu'offre chaque département, le nombre d'étudiants dans chacun de ces cours est, règle générale, assez restreint. Environ quatre-vingts élèves ont suivi le cours de Civilisation canadienne-française depuis 1940, c'est-à-dire une moyenne de dix élèves par année.

Les champs de spécialisation représentés par ces étudiantes offrent une grande variété: civilisation américaine, histoire, économie politique, littérature anglaise, littérature française, art, etc., etc. Cette variété de préparations pose certains problèmes dont l'un, celui de la langue à employer dans le cours, dût être résolu dès 1942. Les deux premières années le cours fut professé en français. La première année tout marcha bien, car la classe était entièrement composée d'étudiantes se spécialisant en français. Dès l'année suivante, des spécialistes en histoire, en économie politique, en civilisation américaine etc, s'inscrivirent pour le cours. En 1942, grâce à l'intérêt et à l'esprit de coopération dont frent preuve certains de mes collègues du département de Civilisation

longue pour être citée ici, il en est de même de celles des Canadiens français et anglais qui ont mis tout en œuvre pour me permettre de me documenter aussi rapidement et aussi complètement que possible. Je mentionnerai quelques noms au cours de cet article. Il faudrait un volume pour les mentionner tous. A tous j'adresse ici mes remerciements les plus sincères, et en particulier, à M. Victor Morin, un ami de toujours, qui m'ouvrit toutes grandes, dès mon premier voyage à Montréal en 1940, non seulement les portes de sa maison mais aussi celles de sa science. 
américaine, notamment le Professeur Daniel Aaron, les étudiantes appartenant à ce département se trouvèrent être la majorité dans le cours de Civilisation canadienne-française. Celles-ci, parfaitement préparées par leurs études, à saisir l'esprit d'une jeune civilisation dont le passé colonial se rapproche du nôtre sur bien des points, ne savaient pas toujours assez de français pour suivre des leçons faites en cette langue, ou même, dans certains cas, pour lire rapidement une foule de textes en français. Dans ces conditions, j'avais ou à faire le sacrifice de recrues qui comptent très souvent parmi mes meilleures élèves et à laisser péricliter le cours, ou à le faire en anglais. J'optai pour cette seconde solution et, depuis 1943, je fais mon cours en anglais. Dans les cas où les étudiantes lisent le français facilement, elles font leurs lectures dans cette langue. Dans les cas contraires, elles lisent des textes anglais. Bien que cette façon de procéder soit loin d'être idéale, elle permet tout de même aux étudiantes de se familiariser avec l'histoire, la littérature, la pensée canadienne-française et avec les problèmes d'ordres divers auxquels les Canadiens français ont à faire face, et petit à petit, à l'aide d'échanges de vues en classe, elles en arrivent à saisir le caractère,le sens et l'importance du fait français en Amérique.

\section{MÉTHODE DU COURS}

Le problème fondamental que présente l'enseignement d'une civilisation étrangère est de rendre celle-ci non seulement vivante mais, surtout, intelligible à l'auditoire auquel cet enseignement s'adresse. Ce problème est encore plus ardu lorsqu'il s'agit d'une civilisation américaine que lorsqu'une civilisation européenne est en cause. Aux Etats-Unis, comme partout ailleurs sur le continent américain, nous connaissons mieux l'Europe que l'Amérique. Nous sommes plus disposés à faire un effort d'esprit pour comprendre une culture européenne que nous ne le sommes pour essayer de saisir les traits distinctifs d'un peuple américain, y compris le nôtre. Cette attitude qui résulte d'une longue tradition est parfaitement naturelle, mais elle n'en est pas moins déplorable. ${ }^{1}$

1. Dans son livre, Reflets d'Amérique (1941), M. Montpetit note cette même tendance dans certaines histoires du Canada où écrit-il: "Il est peu question des Etats-Unis, si ce n'est pour rappeler la guerre de l'Indépendance et en tirer la sa isfaction d'une victoire, et la guerre de 1812. Deux faits qui se réduisent d'ailleurs à décrire les opérations militaires et à énoncer les traités de paix. " 
D'autre part, même si les peuples américains connaissent insuffisamment leur propre histoire, ils en ont inévitablement recueilli quelques bribes et il est toujours possible, surtout lorsqu'il s'agit d'une autre civilisation d'origine coloniale, de partir de certains faits connus pour aller aborder l'inconnu. C'est à ce point de vue que je me place pour introduire dans toute sa force l'idée du Fait fransais en Amérique, et particulièrement au Canada, dans l'esprit de mes étudiantes.

Les premières leçons du cours sont consacrées à la période des grandes explorations européennes en Amérique et à l'établissement des colonies anglaises, espagnoles, françaises et portugaises sur le continent américain. Ici, je m'efforce à mettre en lumière certains parallèles qu'offrent entre elles les civilisations du Nouveau Monde: un passé colonial, la préservation à travers les siècles de certaines traditions fondamentales, l'évolution de la langué, le développement insensible et continu d'un sentiment national, etc. Ces premières leçons, dont l'une est faite chaque année par Mrs. V.L.B. Holmes du département d'histoire, ont pour but de " situer " le Canada français moderne selon les lois de la perspective géographique et historique, c'est-à-dire, une civilisation nord-américaine d'origine et de traditions françaises, coloniales et catholiques; une civilisation qui, en dépit des vicissitudes qui ont marqué le cours de son histoire, a évolué et continue d'évoluer selon les grandes lignes qu'ont suivies les autres peuples américains. Tout en tenant compte du fait extrêmement important que le Canada françaîs fait partie d'un Dominion britannique, j'insiste sur le fait également important que la province de Québec incarne l'aspect moderne de la Nouvelle-France tout comme le Mexique, le Brésil ou les Etats-Unis, par exemple, représentent, à leur état moderne, des traditions transplantées de leur pays d'origine au XVIe et au XVIIe siècles. Etant donné que la période coloniale a laissé une empreinte profonde sur toutes ces civilisations, il va sans dire que les traits caractéristiques de la Nouvelle-France font l'objet de plusieurs leçons.

Afin de mettre en relief non seulement l'histoire de la colonie mais aussi le terrain auquel les premiers colons durent s'attaquer, les ressources qu'ils $\mathrm{y}$ trouvèrent, le milieu physique, enfin, auquel ils durent s'adapter, une de mes collègues du département de géologie, Miss Elizabeth Olmstead dont les recherches personnelles portent sur le Canada, a l'obligeance de venir nous faire une conférence sur le sol 
du Québec. Cette leçon éclaire non seulement le passé en jetant un jour moderne sur l'admirable petit livre de Pierre Boucher, Histoire véritable et naturelle des moeurs et productions du pays de la NouvelleFrance (1663), mais elle prépare aussi les étudiantes à saisir la portée de certains développements miniers actuels, tels ceux du Nouveau Québec.

L'étude de la période coloniale sous autant d'aspects que le permet le temps relativement court dont nous disposons, nous mène jusqu'au début de novembre. Pendant ces premières semaines, les étudiantes se sont familiarisées avec les grands noms de la Nouvelle-France: Jacques Cartier, Samuel de Champlain, Louis Hébert, Pierre Boucher, les martyrs: Brébeuf, Jogues et Lallemant; Mgr. de Laval, Mère Marie de l'Incarnation, Maisonneuve, Marguerite Bourgeoys, Jeanne Mance, La Vérendrye, d'Iberville etc., etc. Grâce à leurs lectures, elles ont une idée sommaire, si l'on veut, mais assez claire des traditions qui sont à la base du Canada français moderne.

D'autre part, il èst très important que la jeunesse d'aujourd'hui se rende compte de l'existence et du caractère du Canada français contemporain. Ainsi, pendant la seconde partie du cours, nous passons en revue rapidement, à l'aide de textes originaux que j'ai fait polygraphier pour nous faciliter la tâche, les débuts du Régime anglais, l'Acte de Québec, la création et l'évolution de la constitution canadienne, le rôle des hommes d'Etat et des publicistes canadiens-français dans cette évolution, le développement de l'instruction publique dans la province de Québec, etc. Ainsi munies de certaines notions d'histoire politique, sociale et intellectuelle, les étudiantes sont prêtes à aborder, avec une certaine compréhension de leur sens véritable, quelques œuvres littéraires canadiennes-françaises. En fait, ces œuvres littéraires rendent aux données historiques ce que celles-ci leur ont prêté. Un résumé d'événements politiques ou sociaux ne prend toute sa signification que lorsque la littérature en a révélé la qualité humaine. Certains passages des Anciens Canadiens ou des Mémoires de Philippe Aubert de Gaspé aident à saisir l'état d'esprit des Canadiens pendant les années qui précédèrent et suivirent de près la Cession. Il en est de même de quelques poèmes de Crémazie ou de Fréchette, si vieux jeu puissent-ils sembler, quand on les envisage d'un point de vue purement esthétique. De même, les œuvres contemporaines reflètent divers aspects de la mentalité canadienne-française, soit par les personnages 
qui sont mis en scène, soit par le point de vue que l'auteur révèle consciemment ou inconsciemment dans ses œuvres: poèmes, romans, essais. Une manifestation de la littérature contemporaine canadienne que je n'ai pas cru devoir omettre comporte les œuvres écrites en vue de la radio. Certaines scènes du Curé de village ou de la Pension Velder de Robert Choquette, certains disques d'Un homme et son péché de Grignon aident à donner du moins une idée du caractère psychologique de certains romans-fleuves radiophoniques dans la Province de Québec.

Toujours dans le but de ne point isoler les diverses manifestations de la civilisation canadienne-française et toujours dans le but de combattre ce mythe grotesque d'un " quaint old Quebec where nothing ever changes " et afin de démontrer que cette civilisation s'est développée d'une façon parfaitement normale, bien que dans des conditions anormales, une de mes collègues du département d'espagnol, Miss Helen Peirce, professeur de littératures sud-américaines, nous fait, chaque année, lorsqu'elle n'est pas au Mexique, au Brésil ou en Argentine, une leçon sur une littérature sud-américaine au cours de laquelle elle est inévitablement amenée à parler des problèmes esthétiques et linguistiques que les auteurs américains de langue espagnole ou portugaise ont eu à résoudre dans l'élaboration de leurs œuvres. J'ai bon espoir que cette année, M. Daniel Aaron nous parlera de la littérature américaine en se plaçant au même point de vue. Bien entendu, le but de ces diverses comparaisons n'est pas de faire entendre que les littératures américaines sont identiques, mais plutôt de souligner la ressemblance qui existe indubitablement entre les obstacles que les jeunes littératures de ce continent ont eu à surmonter et ont encore à surmonter pour arriver au rang où elles aspirent. Sauf du point de vue des influences, la comparaison de la littérature canadienne-française à la littéra ture française est absurde. Cette comparaison repose sur des données qui n'ont aucun sens. Il en est de même pour toutes les littératures de notre continent. On ne peut, dans leur ensemble, les comparer à celle de leurs mères-patries respectives. Il ne s'agit pas de démontrer que la littérature canadienne-française est de premier ou de dernier ordre, ou d'affirmer qu'elle est belle parce qu'elle est canadienne-française. Il s'agit encore moins, si possible, de la regarder d'un œil attendri ou apitoyé. Il s'agit tout simplement de l'envisager telle au'elle est: une jeune littérature qui se développe, non point à coups 
de chefs-d'œuvre, mais grâce à l'effort soutenu de générations successives; une jeune littérature qui commence actuellement à refléter de façon de plus en plus frappante la mentalité et les problèmes du milieu national et social dont elle émane.

Un des aspects les plus importants du cours de Civilisation canadienne-française est le travail de longue haleine que chaque étudiante doit remettre à la fin du semestre, avant les derniers examens. Comme je le disais au début de cet article, les étudiantes de ce cours sont en train de se spécialiser dans une grande variété de sujets. Ce manque d'uniformité dans la préparation qu'elles apportent à un cours qui dépasse lui-même le cadre des conventions établies par la tradition académique, peut sembler, au premier abord, quelque peu décourageant, D'autre part, et pour peu qu'on y regarde de près, cette variété de préparation possède aussi certains avantages. Une spécialiste en économie politique, en histoire, en art ou en littérature est parfaitement préparée à fournir un travail à la fois solide et intéressant sur un sujet canadien se rattachant au champ d'étude dans lequel elle se spécialise. L'expérience m'a appris que la meilleure façon de permettre aux étudiantes de se rendre pleinement compte de la portée du fait français en Amérique est de leur laisser le choix d'en étudier une phase qui se rattache soit à leur propre spécialité, soit à leur expérience personnelle. Après avoir consacré deux mois à des recherches personnelles sur une manifestation quelconque de l'activité française en Amérique, les étudiantes ont une idée beaucoup plus claire de l'apport des Français au développement du continent américain qu'elles n'en auraient si le travail du cours s'était borné pour elles à suivre des conférences, à écrire un ou deux devoirs et à passer un examen. La méthode que j'ai adoptée et qui consiste à encourager les étudiantes à fournir un travail personnel, tout en assimilant des connaissances nouvelles, n'a rien de très original, mais elle se prête d'une façon particulièrement heureuse à un cours comme French $312 a$ dont la matière à peu près inconnue ic $i$ se rattache néanmoins de très près à notre histoire. Quelques titres de travaux présentés au cours de ces dernières années donneront une idée de leur nature.

\section{L'art au Canada français.}

Un travail sur cet aspect de la civilisation canadienne-française est présenté chaque année par une étudiante, sous la forme d'une confé- 
rence illustrée qui a lieu chez moi, dans la soirée. Grâce à la générosité du Secrétariat de la Province et en particulier de M. Gérard Morisset, nous disposons d'une fort belle collection de projections qui inclut des exemples d'architecture, de sculpture et d'orfèvrerie de l'ancien régime. De plus j'ai recueilli moi-même au Musée National à Ottawa, plusieurs projections qui illustrent la peinture moderne au Canada. A l'aide de ces projections ainsi que des livres de M. Morisset et des articles publiés par MM. C. Marius Barbeau et Ramsay Traquair sous les auspices de l'université McGill, les étudiantes préparent leur conférence.

Le Folklore canadien-français.

Travail présenté en 1941 par une étudiante qui savait admirablement le français et qui habitait Washington: ce qui lui permit d'avoir recours, pendant ses vacances, au remarquable département de Folklore de la Bibliothèque du Congrès.

L'œuvre poétique de Paul Morin.

Travail présenté par une spécialiste de français en 1941.

Le roman canadien-français.

Un travail sur ce sujet est présenté chaque année par une étudiante sachant bien la langue française.

Le Bloc populaire.

Travail présenté en 1944 par une Anglo-canadienne de Montréal, spécialiste d'histoire.

Le système d'Instruction publique dans la province de Québec.

Travail présenté par une Anglo-Canadienne de Toronto, spécialiste d'économie politique. Cette étudiante a obtenu de ce département la permission de consacrer une partie de son temps, l'an prochain, à un travail sur l'évolution du sentiment national au Canada.

Parmi les étudiantes qui viennent de l'ouest des Etats-Unis, plusieurs ont remis des travaux sur les origines françaises de leur Etat 
natal: Indiana, Illinois, Missouri, Wisconsin, Minnesota et, naturellement, la Louisiane. J'ai eu récemment l'occasion de causer avec une ancienne étudiante dont les recherches avaient porté, il y a trois ans, sur le Wisconsin. Elle est actuellement journaliste à Milwaukee et elle venait d'être chargée de la rédaction d'une série d'articles sur l'histoire du Wisconsin.

Ces travaux sur l'histoire des Etats dont les étudiantes sont originaires, bien qu'ils ne touchent qu'indirectement à la matière principale du cours, s'en éloignent moins qu'on ne pourrait le croire à première vue. Ces travaux, en familiarisant leurs auteurs avec leur propre milieu, les convainquent d'une façon très personnelle de l'importance du fait français sur le continent américain. Il est à noter que, dans la majorité des cas, les étudiantes qui choisissent ce genre de sujet pour leur travail personnel, sont spécialistes de civilisation américaine. Les recherches qu'elles font pour mon cours se rattachent, par conséquent, d'une façon très étroite à leur champ de spécialisation. D'autre part, les conférences auxquelles elles assistent au cours du semestre, les lectures qu'elles font, les discussions auxquelles elles prennent part, élargissent leur horizon et étoffent leurs connaissances.

Les dernières leçons du cours sont consacrées aux Canadiens français en dehors du Québec et aux Franco-Américains de la NouvelleAngleterre. En 1941, M. Edward Ham qui était alors à Yale et qui est actuellement à l'université du Michigan voulut bien venir nous faire une conférence sur ce groupe auquel il consacre depuis plusieurs années une bonne partie du temps que lui laissent ses recherches professionnelles. M. Ham, co-fondateur du groupe North American French Language and Literature de la Modern Language Association, est président de ce groupe. Il a succédé à M. William Locke, chef du département des langues modernes au Massachusetts Institute of Technology, auteur d'une étude de grande valeur sur les FrancoAméricains de Brunswick, Maine, et dont certaines parties ont été publiées dans les Archives de Folklore. J'espère toujours pouvoir l'attirer ici un jour pour une conférence, ainsi que M. Adolphe Robert, président général'de l'Association canado-américaine à qui je suis redevable d'une foule de renseignements précieux sur les Franco-Américains. M. Robert, avec la courtoisie que tous lui connaissent, a mis à ma 
disposition la Collection Lambert dont l'organisation qu'il dirige est propriétaire.

Comme on peut en juger par l'aperçu qui précède, la méthode adoptée pour l'enseignement de la civilisation canadienne-française est extensive plutôt qu'intensive. Elle résulte en partie de la richesse de la matière à inculquer, en partie de la variété de préparations que présentent les étudiantes ainsi que des intérêts divers qui les engagent à suivre ce cours. Le but que je me suis proposé en l'adoptant, après quelques années de tâtonnement, est de faire jaillir l'étincelle qui puisse allumer chez mes élèves le désir d'étudier et de comprendre le fait français en Amérique, non point comme quelque chose qui leur est étranger, mais comme un élément important et vivace de l'Amérique moderne.

Cette méthode, telle qu'elle existe aujourd'hui, me semble donner des résultats satisfaisants. Evidemment, je ne voudrais pas avoir l'air de vouloir affirmer qu'elle ne pèche point par certains côtés. Toutefois, elle a ceci de bon qu'envisagée du point de vue des élèves, elle contribue à éveiller chez elless un intérêt personnel dans le sujet qu'elles étudient; et qu'envisagée du point de vue du professeur, elle est susceptible d'améliorations continuelles.

\section{LA BIBLIOTHÈQUE: COLLECTION CANA DIENNE}

Il va sans dire que le cours de civilisation canadienne-française ne saurait exister au niveau universitaire s'il ne reposait sur une solide bibliothèque canadienne. En 1940, à l'époque où j'organisais ce cours, je ne disposais d'aucun budget spécial pour l'achat de livres canadiens. Il se trouva que, précisément à cette date, le département de langue et de littérature française à Smith College était dans l'impossibilité d'importer des livres de France. Il m'autorisa donc, en tant que membre du département, à consacrer une partie de notre budget à l'achat de livres canadiens-français. Ceci me permit de jeter les fondements d'une bonne collection canadienne, avec l'aide de bibliophiles tels: Victor Morin, Aegidius Fauteux et G. Ducharme, au Canada, et de Miss Mary Dunham, ancienne conservatrice de la Bibliothèque à Smith College dont la collaboration et les encouragements me furent extrêmement précieux. A partir de 1943, l'administration de Smith College m'octroya un budget spécial grâce auquel il m'est possible d'acquérir, 
chaque année, un certain nombre de volumes canadiens en français et en anglais et de souscrire à une douza ine de revues et de journaux canadiens tels: La Revue Dominicaine, La Nouvelle Relève, l'Action Nationale, Relations, Liaison, Gants du Ciel, Les Archives de Folklore, La Revue de l'Université Laval, La Revue d'Histoire de l'A mérique Française, Vie Française, Les Transactions de la Société Royale du Canada, Hansard, Le Devoir, Le Canada, Le Travailleur, The Montreal Gazette, The Toronto Globe and Mail, Saturday Night, The Canadian Forum etc.

Deux principes m'ont guidée dans l'organisation de notre collection canadienne. Premièrement, cette collection doit avoir pour but de fournir une vue d'ensemble de l'activité canadienne-française dans une foule de domaines: histoire, art, géographie, science, industrie, commerce, études sociales, religion, pédagogie, musique, folklore, littérature etc. Deuxièmement, j'ai insisté particulièrement pour que notre collection ne soit point placée sur nos rayons à titre de collection spéciale et pour, qu'au contraire, les volumes qui la composent soient disséminés dans toutes les sections de la bibliothèque, selon les sujets qu'ils traitent. Ainsi, les livres d'histoire se trouvent dans la section réservée aux études historiques. Ceux qui traitent de géographie, de littérature, de religion ou de sociologie, font partie des sections réservées à ces sujets. Outre que cette façon de procéder est pratique du point de vue bibliothéconomique, elle offre l'avantage d'attirer l'attention des membres du collège sur l'activité intellectuelle canadiennefrançaise. La Bibliothèque de Smith College compte plus de 350,000 volumes et on y a toujours pratiqué le système dit des " Open Shelves " où professeurs et étudiantes ont le droit de bouquiner à leur aise parmi les rayons. Il m'a semblé qu'il y avait tout avantage pour les écrivains canadiens à ce que leurs œuvres soient placées dans les sections auxquelles elles se rattachent par le sujet plutôt qu'à les voir reléguées dans une collection spéciale assez peu accessible à la moyenne des lecteurs qui seraient ainsi privés de l'occasion d'utiliser toutes les ressources dont dispose la bibliothèque. D'autre part, ce que j'espère, c'est d'obtenir un jour, les fonds nécessaires qui nous permettraient d'inviter une bibliothécaire canadienne-française à venir ici établir un ca talogue raisonné de notre collection canadienne. Celui-ci serait séparé du catalogue général et constituerait un instrument de travail fort utile pour les spécialistes. 
Dans la situation actuelle, il est assez difficile d'indiquer avec précision le nombre de volumes que comporte la collection canadiennefrançaise de Smith College. Ce nombre s'élève, sans doute aucun, à plus de trois mille volumes parmi lesquels on trouve certaines raretés qui sont naturellement placées sur les rayons de la Rare Book Room: La Vie de la Vénérable Mère Marie de l'incarnation, Paris, 1677; L'Histoire de la Nouvelle-France de Charlevoix, Paris, 1744; Lafitau, Mœurs des sauvages amériquains, Paris, 1724-32 etc.

Parmi les instruments de travail indispensables, la bibliothèque possède: L'Essai de bibliographie canadienne de Philéas Gagnon, le Catalogue d'ouvrages sur l'Amérique de G.-B. Faribault, le Dictionnaire généalogique de Tanguay, les œuvres de Henry Harrisse, The Canadian Catalog of Books published in Canada de la Public Library de Toronto ainsi que la bibliographie d'œuvres canadiennes publiées par l'université McGill: le Dictionnaire Général du Père LeJeune, l'Encyclopedia of Canada de W. Stewart Wallace, etc., etc., etc. La bibliothèque possède également une série très complète des documents publiés par les Archives publiques à Ottawa et des documents publiés par la Province de Québec. Les premiers se trouvaient ici avant 1940. C'est à M. Antoine Roy que nous sommes redevables des seconds.

Dans la section d'histoire se trouvent naturellement les Oeuvres de Champlain (édition Laverdière), celles de Michel Bibaud, de F.-X. Garneau, de Faillon, Ferland, Casgrain, Gérin-Lajoie, A. Gosselin, Benjamin Sulte, L.-O. David, Joseph-Edmond Roy, Sir Thomas Chapais, Pierre-Georges Roy, le Chanoine Groulx, l'Abbé Tessier, Gustave Lanctot, Jean Bruchési, J.-N. Fauteux, G. Filteau, Salone, l'abbé Maheux, Robert Rumilly, Guy Frégault, Léo-Paul Desrosiers, etc., etc. Parmi les historiens canadiens de langue ang]aise: George M. Wrong, C.W. Colby, A.L. Burt, D.G. Creighton, A.R. Lower, Edgar McInnis, J.B. Brebner, sans parler des éditeurs de séries telles: Canada and its Provinces, The Makers of Canada, Chronicles of Canada, ou de publications en français comme celles de la Société historique de Montréal, et, dans un autre genre, celles de la société des Dix.

Quant à la littérature canadienne-française, notre collection est très riche. On y trouve, bien entendu, les œuvres de Philippe-Aubert de Gaspé, de Crémazie, de l'abbé Casgrain, ainsi que des recueils comme Le Répertoire national de Huston, Le Foyer Canadien, Les soirées canadiennes, Les nouvelles soirées canadiennes, Les Guêpes, La 
Lanterne, La Revue canadienne, L'Ordre de Olivar Asselin, La Revue trifluvienne. Notre collection d'auteurs canadiens-français contemporains est à peu près complète et je m'efforce de la tenir à jour.

A la discothèque du Département de musique, on trouve une soixantaine de chansons de folklore canadiennes qui ont été enregistrées dans les Etats d'Indiana et de Michigan par Alan Lomax, alors qu'il était attaché à la section de Folklore de la Bibliothèque du Congrès à Washington. Cette collection est intéressante, mais nous attendons avec impatience celle des enregistrements de Marius Barbeau dont les rouleaux et disques originaux se trouvent actuellement à Ottawa.

A titre de documents contemporains, à l'usage de l'avenir, j'ai ajouté à cette collection certains disques populaires canadiens-français achetés à Holyoke, Massachusetts, tel, Camillien nous revient. D'après les renseignements obtenus à Holyoke, le chiffre de disques de ce genre importés du Canada chaque mois s'élève à plusieurs milliers. Bien que je n'aie point encore utilisé ces disques dans mon cours, je leur trouve personnellement un certain intérêt sociologique. Le répertoire des cafés-concerts reflète à sa manière les joies et les préoccupations du peuple tout comme le folklore de jadis.

\section{ACTIVITÉs.}

Il n'est peut-être pas déplacé de mentionner ici, certaines activités qui, si elles ne font pas partie intégrante du cours de civilisation canadienne-française, n'en ont pas moins contribué puissamment à l'enrichir et à le vivifier.

En 1941, M. Victor Morin passait deux jours à Smith College et faisait une conférence qui fut fort appréciée sur le folklore canadienfrançais. En 1942, M. Robert Choquette était nommé Resident Author à Smith College, sous les auspices de la Rockefeller Foundation. Au cours de l'année qu'il passa ici, il fit plusieurs causeries dont l'une au Faculty Club de Smith College et deux autres au cours de Civilisation canadienne-française. Il prêta généreusement ses lumières au cours de Script Writing que le département d'art dramatique était alors en train de mettre sur pied. Le charme de sa sympathique présence se fit sentir dans tout le collège.

En 1943, le Musée d'art de Smith College exposait pendant quinze jours la magnifique collection de peintures modernes cana- 
diennes qu'avaient recueillie au Canada, avec la coopération de Mademoiselle Marie Maréchal, M. Bartlett Hayes, directeur du musée d'art de Phillips Academy, Andover, Massachusetts et M. Patrick Morgan, professeur d'art à cette même institution.

En 1944, M. Harry Bernard, alors en voyage d'études aux EtatsUnis sous les auspices de la Rockefeller Foundation, fit un bref séjour à Northampton au cours duquel il adressa la parole à French 312 a et fut reçu au Faculty Club où il rencontra plusieurs membres du département de civilisation américaine. Cette même année, le Professeur Frank Scott de McGill University, invité par le département d'histoire a faire trois conférences sur le Canada, nous parla avec chaleur et intelligence du Canada français.

En 1945, quatre des étudiantes qui avaient suivi le cours de Civilisation canadienne-française organisèrent un voyage de dix jours au Canada et m'invitèrent à me joindre à elles. Nous fûmes reçues à Montréal, aux Trois-Rivières, à Québec et à Ottawa avec la plus grande cordialité. A Montréal, les étudiantes furent émerveillées de la splendeur de lU'niversité. Le même jour, nous visitions l'École du Meuble et M. Jean-Marie Gauvreau nous recevait à un déjeuner Chez Pierre auquel assistait MM. Victor Barbeau, Maurice Gagnon, Gérard Morisset et le regretté Marcel Parizeau.

Le lendemain, M. Léo-Paul Desrosiers, conservateur de la Bibliothèque de Montréal, nous fit visiter la collection Gagnon que mes élèves connaissaient déjà de réputation, et nous offrit un thé que présida Michelle LeNormand et où les étudiantes purent causer avec les membres de la bibliothèque. M. J.-J. Lefebvre, alors conservateur de la Bibliothèque St-Sulpice, nous fit également les honneurs de cette bibliothèque et $M$. Robert Choquette ceux de Radio-Canada. Grâce à l'initiative de Mme Gérard Lortie et à l'hospitalité du directeur du Collège Stanislas, les étudiantes purent assister à certaines classes à cette institution et voir des écoliers canadiens-français à l'œuvre et à l'épreuve.

Aux Trois-Rivières, l'abbé Tessier nous reçut dans son bureaumusée avec la cordialité qui lui est coutumière. Après avoir visité deux industries du papier de fond en comble, sous la direction des ingénieurs de deux compagnies, nous partions pour Québec où nous avions le plaisir de retrouver l'abbé Tessier qui se joignit à nous pour une expédition à Saint-Jean-Port-Joli et à Sainte-Anne de la Pocatière 
qu'avait organisée à notre intention Mademoiselle Evelyne Leblanc avec le concours du département de l'Agriculture. A Québec où les élèves surent apprécier le caractère français et historique de l'Université Laval, l'abbé Parent nous reçut à dîner au Cercle universitaire et le Père G.-H. Lévesque, Doyen de la Faculté des Sciences sociales, présenta mes étudiantes à plusieurs des siens qui leur offrirent un thé a la Maison des Etudiants et mirent tout en œuvre pour nous rendre le séjour dans la vieille capitale aussi instructif qu'il était agréable. Comme le parlement siégeait au moment de notre passage à Québec, il nous fut possible d'assister à une séance. Nous fûmes aussi reçues par les officiers de la Citadelle.

A Ottawa, M. Gustave Lanctot, Directeur des Archives, consacra généreusement une bonne partie de la matinée à nous faire voir et comprendre les précieux documents dont il est le dépositaire. Au Musée National, M. McCurrie se chargea lui-même de nous faire visiter la collection de peintures canadiennes qui n'était pas exposée dans les salles publiques au moment de notre voyage. M. Marius Barbeau, que les étudiantes désiraient beaucoup rencontrer, était malheureusement en dehors de la ville. Au parlement, nous eûmes la chance de trouver M. Louvigny de Montigny dans son bureau. Les étudiantes le connaissaient également par son livre sur Maria Chapdelaine et par le rôle qu'il joua dans la publication de ce chef-d'œuvre. Avec un sens de l'hospitalité authentiquement canadien, il s'offrit à nous faire visiter le parlement lui-même et il enrichit cette visite de ses commentaires historiques.

En 1947, Miss Doris Silbert, exquise pianiste et musicographe avertie du département de musique à Smith College, organisait une soirée de chansons canadiennes à la Maison Française. Ce fut une soirée inoubliable à laquelle assistaient près de cent cinquante personnes, professeurs et étudiantes. Après avoir expliqué et illustré au piano la composition de chansons très anciennes: Le prince Eugène, Le Prince d'Orange, La sainte vierge aux cheveux pendants, Les trois roses empoisonnées, Voici le printemps, et, bien entendu, Ysabeau s'y promène, $E n$ roulant ma boule et $A$ la claire fontaine, Miss Silbert qui avait déjà consacré quelques heures à entraîner les étudiantes du cours de civilisation canadienne-française à chanter ces mélodies dont certaines sont si complexes pour nos oreilles modernes, encouragea l'auditoire qui avait en main les paroles de ces chansons à se joindre au groupe 
déjà entraîné et à chanter et à rechanter ces splendides mélodies d'un autre âge. Ce fut une révélation pour nous tous.

L'année 1948 s'annonce bonne. Douze étudiantes sont inscrites pour le cours de 1948. Le directeur du Musée d'art à Smith College est en pourparlers avec $M$. Patrick Morgan dans le but d'avoir ici une exposition de la collection personnelle d'œuvres de peintres et sculpteurs dits " primitifs " que $\mathbf{M}$. Morgan a recueillie avec gout et intelligence lors de ses nombreux séjours dans le comté de Charlevoix. Nous espérons aussi faire venir d'Ottawa un nombre suffisant de documentaires pour constituer un programme complet sur le Canada. Enfin, nous entretenons toujours l'espoir qu'un Canadien ou une Canadienne de passage dans les environs veuillent bien venir ici nous parler de sa spécialité.

En somme, bien que la tâche accomplie jusqu'ici à Smith College soit modeste et bien qu'il reste encore énormément à faire, il est permis d'envisager l'avenir avec confiance. $\mathrm{Si}$, comme je le disais au début de cet article, les pays américains n'en sont pas encore arrivés à se connaitre les uns les autres comme ils le méritent, ils s'acheminent néanmoins vers cette connaissance; et tout porte à croire que d'ici quelques années on notera des progrès considérables sous ce rapporrt. Pour ma part, l'expérience des huit dernières années m'engage à affirmer que nous sommes sur la bonne voie.

Marine Leland, Ph.D.

Professeur de littérature française et de civilisation canadi nne-française.

SMITH COLLEGE

Northampton, Massachusetts. 